\title{
Erratum: Noncanonical roles of membranous Iysyl-tRNA synthetase in transducing cell-substrate signaling for invasive dissemination of colon cancer spheroids in 3D collagen I gels
}

\section{Seo Hee Nam, Doyeun Kim, Mi-Sook Lee, Doohyung Lee, Tae Kyoung Kwak, Minkyung Kang, Jihye Ryu, Hye-Jin Kim, Haeng Eun Song, Jungeun Choi, Gyu-Ho Lee, Sang-Yeob Kim, Song Hwa Park, Dae Gyu Kim, Nam Hoon Kwon, Tai Young Kim, Jean Paul Thiery, Sunghoon Kim and Jung Weon Lee}

Present: Due to a technical error during publication, duplicate videos were uploaded as Supplementary File 5 and Supplementary File 28. As a result, Figure 5E contains a reference to the duplicate files.

Corrected: Correct Supplementary File 28 was uploaded. Updated figure 5E can be found below. The publisher apologizes for this oversight.

Original Article: Oncotarget. 2015; 6: 21655-21674. doi: 10.18632/oncotarget.4130.

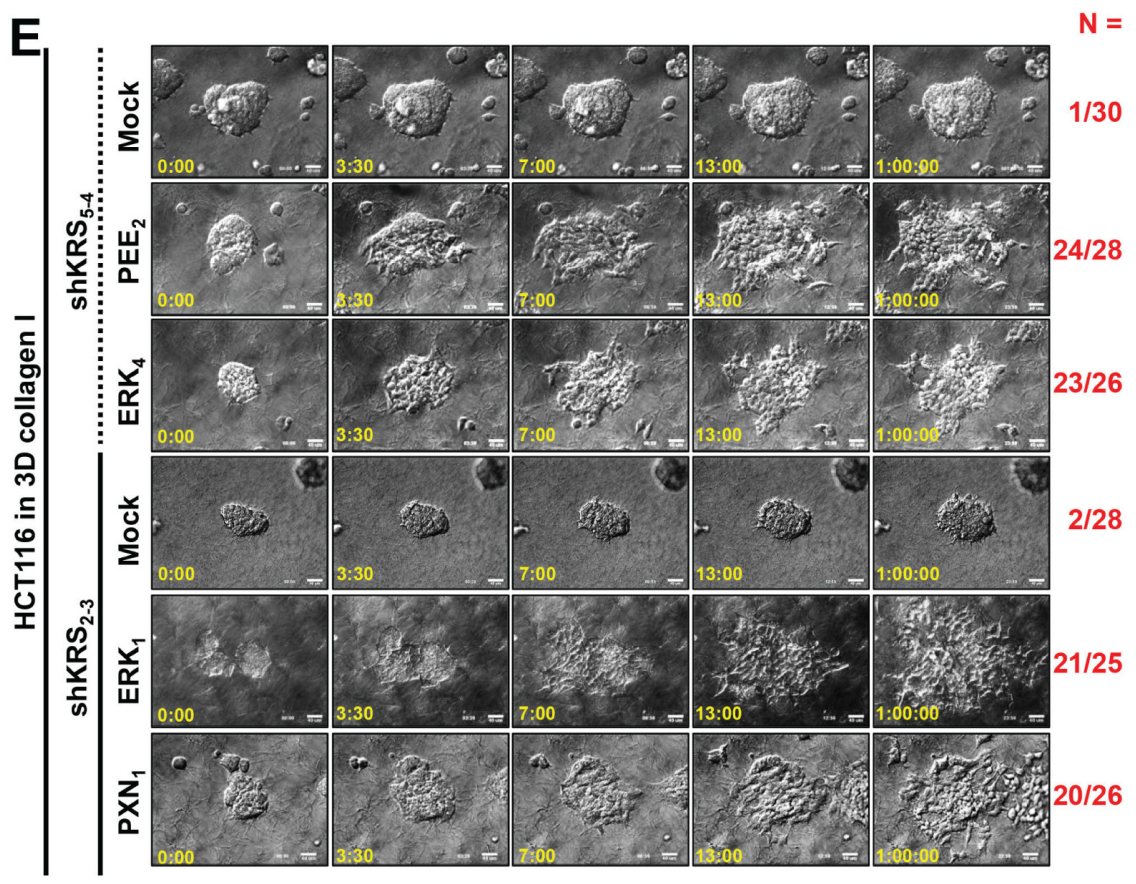

Figure 5: Blockade of dissemination of KRS-suppressed cells was relieved by ERK1/2 and/or paxillin expression. (A) HCT116 parental cells were suspended in serum-free BSA containing media, preincubated with normal IgG or functional anti-human integrin $\alpha 6$ blocking antibody $(20 \mu \mathrm{g} / \mathrm{ml})$ for $30 \mathrm{~min}$, and then kept in suspension or reseeded onto lamininprecoated culture dishes with $2 \%$ serum-containing media for 2 or $24 \mathrm{~h}$. Whole-cell lysates were immunoblotted for the indicated molecules. (B and C) Cells were transduced with adenovirus (Ad-HA) for R454 (kinase dead) FAK, $\triangle \mathrm{N}(1-100)$ FAK, or FAK WT for $12 \mathrm{~h}$. The cells were embedded in 3D collagen I gels and, $3 \mathrm{~h}$ later, the cell lysates were prepared for immunoblotting (B) or time-lapse imaging was performed for a further $28 \mathrm{~h}(1: 04: 00, \mathrm{C})$. The numbers to the right of the figure in the ' $\mathrm{a} / \mathrm{b}$ ' format depict disseminative phenotype cases/total experimental cases. See also Movie S17 to 24. In anti-HA antibody blot, the exogenous FAK showed two different sizes due to WT and its N-terminal deletion, but FAK phosphorylation blots appeared to be single band due to robust phosphorylations mediated by the N-terminal deletion mutant FAK [29]. (D and E) KRS-expressing cells and KRS-suppressed HCT116 cell spheroids (21, 2-3 and 5-4 clones) with stable transfection of ERK1/2 (ERK), paxillin (PXN), or ERKs/paxillin (PEE) were embedded in 3D collagen I gels for $24 \mathrm{~h}$, prior to harvesting whole-cell lysates for immunoblottings (D). Alternatively, the spheroids were embedded for $3 \mathrm{~h}$ and then time-lapse imaged for another $24 \mathrm{~h}$ (E). See also Movie S25 to 30. Data represent three independent experiments. 